\title{
Flow Curves in the Centered Cylindrical Couette Geometry of a Polyethylene Oxide Solution
}

\author{
Ngargoto Ngarmoundou',2, Rilengar Godfroyd Ousman1,2, Barka Mahamat ${ }^{3}$, \\ Aboubaker Chedikh Beye ${ }^{1}$ \\ ${ }^{1}$ Group of Laboratories of Physics of Solids and Materials Science (GPSSM), Department of Physics, Faculty of Sciences and \\ Techniques, Cheikh Anta Diop University, Dakar, Senegal \\ ${ }^{2}$ Department of Mechanic, Faculty of Sciences and Techniques, University of Mongo, Mongo, Chad \\ ${ }^{3}$ Department of Physical Science, Faculty of Exact and Applied Sciences, University of N'Djamena, N'Djamena, Chad \\ Email: nngarmoundou@yahoo.fr
}

How to cite this paper: Ngarmoundou, N., Ousman, R.G., Mahamat, B. and Beye, A.C. (2017) Flow Curves in the Centered Cylindrical Couette Geometry of a Polyethylene Oxide Solution. Open Journal of Fluid Dynamics, 7, 673-695.

https://doi.org/10.4236/ojfd.2017.74044

Received: November 21, 2017

Accepted: December 26, 2017

Published: December 29, 2017

Copyright (C) 2017 by authors and Scientific Research Publishing Inc. This work is licensed under the Creative Commons Attribution International License (CC BY 4.0).

http://creativecommons.org/licenses/by/4.0/

\begin{abstract}
Polyethylene oxide solutions have a behavioral flexibility that provides researchers with the opportunity to use constitutive law models in a variety of ways for their MRI characterization. Our results obtained in numerical simulation carried out in 2D and 3D for speed profiles of a solution of PEO deployed by the simple method of the cylindrical Couette geometry considering the fluid Newtonian defect, allowed to identify the behavior of fluid complex (rheo-fluidifying threshold fluid). The relevance and the interest of the method are examined by analyzing these results generated by the numerical data obtained, since these profiles depend on the non-Newtonian properties of the fluid which one does not know a priori and which one seeks to measure by postulating first to the power law of Ostwald, then to the truncated power law.
\end{abstract}

\section{Keywords}

Rheology Behavior, Polyethylene Oxide, Couette Geometry, Viscosity, Speed Profiles, Shear Stress, Shear Bands, Shear Rate, Localization, Rheo-Fluidifying Threshold

\section{Introduction}

It is well known that the use of an industrial product of any kind always requires the use of materials in generally liquid, pasty or solid states with a choice based on the physical, chemical, mechanical and rheological properties obtained by experimental means. Natural polymers or synthetic polymers, with very maneuverable properties, are no longer a scarce commodity and their uses are wide- 
spread and varied in industrial sectors, basic academic research and in the commercial sector; the case of polyethylene oxide has better advantages because of its low cost and its behavioral flexibility. For example, PEO is used as an additive in polymerization reactions to prevent foaming and promote polymerization (in the production of vinyl chloride and acrylonitrile butadiene styrene) [1], or for spinning nanofibers.

The rheological properties of polyethylene oxide solutions are related to the concentration, molecular weight, nature of the solvent, external parameters (temperature, pressure) and microstructural interactions (polymer-solvent or polymer-polymer) [2] [3], and on the other hand that the polyethylene oxide is a rheo-fluidifying or rheo-thickening fluid with threshold in steady state [2]-[10].

In our study, we used Couette geometry to simulate and evaluate the non-uniform flow curves of the PEO solution at an entanglement concentration. We then study the rheological behavior of this solution in order to convert the imposed or measured experimental quantities of torque and rotational speed into Couette geometry in the form of a shear rate constraint relationship. The process initiated from the Newtonian model used by default, continues with the Oswald law model (which has remarkable shortcomings in not taking into account the localization observed when the solution is flowed at low velocities of rotation since the existence of a flow threshold is not predicted by this model) and then to the model of the truncated power law. In particular, we would like to show that the use of the Reynolds number $R_{e}$ which is the main parameter governing the different types of fluid flows in the Couette cylindrical geometry is possible.

\section{Materials and Equipment}

The sample of the material used in this study is a solution based on polyethylene oxide (PEO) with a molar mass of $106\left(\mathrm{~g} \cdot \mathrm{mol}^{-1}\right)$ and a concentration of $1.8 \% \mathrm{wt}$. $\mathrm{PEO}$ is a linear chain nonionic polymer $\left[\mathrm{CH}_{2}-\mathrm{CH}_{2}-\mathrm{O}\right] \mathrm{n}$. The choice is made on the geometry of Couette with imposed shear (i.e. we measure the torque on the inner cylinder after having imposed its rotational speed $\omega=2 \pi N(\mathrm{rd} . / \mathrm{s})$, of the coaxial cylinders type with wide gap in front of the microstructure size to avoid the effect of size which makes the smooth surface of cylinder $R_{1}$ sensitive to slippage, hence its coating sometimes with the Emeri canvas. The shear in the geometry is considered homogeneous, which can ensure that the material has the same structure throughout the air gap. The two coaxial cylinders are of inner radius $R_{1}=13.375(\mathrm{~mm})$ and rotates at an angular velocity of 0.002 to 0.9 (rd./s), resulting in low rotational speeds of 0.0026 to $1.20(\mathrm{~cm} / \mathrm{s})$ and shear ratio of 0.2 to $96\left(\mathrm{~s}^{-1}\right)$. The cylinder $R_{1}$ with height $h=40.12(\mathrm{~mm})$ ensures sufficient contact to increase the torque. The outer radius $R_{2}=13.55(\mathrm{~mm})$ is fixed, the curvature $R_{1} / R_{2}=0.99$. The numerical simulations of the velocity profiles are carried out with the software MATLABR2008b. The conversion of the macroscopic data into a behavior law requires an adapted analysis. 


\section{Rheological Study in Steady State}

\subsection{Speed Profile for a Newtonian Fluid in a Cylindrical Couette}

The first work is based on the determination of the shear rate on which the constituent laws predominantly lead to the equations of the velocities. In cylindrical coordinates, this requires knowledge of the speed gradient from which they derive [11]. Regardless of the laminar flow fluid in the Couette cylindrical geometry, the shear rate depends on the tangential velocity component $V_{(\theta, r)}(r)$ expressed by Equation (1) [12].

$$
\dot{\gamma}=\frac{\partial V_{(\theta, r)}(r)}{\partial r}-\frac{V_{(\theta, r)}(r)}{r}=r \frac{\partial}{\partial r}\left(\frac{V_{(\theta, r)}}{r}\right)
$$

The search for the velocity profile under the multiple flow hypotheses is done as follows:

1) Laminate which imposes a low speed for a displacement in layer of lamellae with respect to each other causing friction forces;

2) Isothermal or permanent to express the independence of variables over time;

3) Incompressible;

4) In the cylindrical coordinate system $(r, \theta, z)$ where the components of the axial, tangential and radial velocities are respectively $V_{z}, V_{(\theta, r)}, V_{r}$, taking into account the symmetry of the problem with respect to $z$ and at $\theta$ and in the absence of an axial pressure gradient, $V_{z}=0$ and $V_{(\theta, r)}$ is independent of $\theta$,

5) The forces of inertia are at all points and at all moment's negligible vis-à-vis the forces of viscosity, which is expressed by: $R_{e} \ll 1$.

The constitutive equation which connects the stress to the shear rates is given by Equation (2):

$$
\tau_{(\omega, r)}=\eta \dot{\gamma}(r)
$$

This shear rate is derived from the expression of the velocity given by Equation (3):

$$
V_{(\omega, r)}(r)=\frac{\omega R_{1}^{2}}{R_{2}^{2}-R_{1}^{2}}\left(-r+\frac{R_{2}^{2}}{r}\right)
$$

or:

$$
\left\{\begin{array}{l}
V_{(\omega, r)}\left(R_{e}, r\right)=R_{e} K_{N G A}\left(-r+\frac{R_{2}^{2}}{r}\right) \\
\text { with: } \\
K_{N G A}=\frac{\mu R_{1}}{\left(R_{2}+R_{1}\right) e^{2}}
\end{array}\right.
$$

$K_{N G A}$ is the period of fluid flow in the air gap in $\left(\mathrm{s}^{-1}\right)$.

The Equation (4) reformulates the Equation (3) in consideration of hypotheses (5), so that the flow velocity takes into account the property of the solution 
related to the Reynold number $R_{e}=\left(\omega R_{1} e\right) / \mu$ with $\mu=\eta / \rho$, the angular velocity $\omega(\mathrm{rd} . / \mathrm{s})$ and the geometry of the duvet outlet device having internal radii $R_{1}$ and outer $R_{2}$. The resulting shear rate is given by Equation (5):

$$
\dot{\gamma}(r)=\frac{2 \omega R_{1}^{2} R_{2}^{2}}{R_{2}^{2}-R_{1}^{2}} \frac{1}{r^{2}}
$$

Thus the stress at the inner cylinder $R_{1}$ or at any radial position $r_{c} \mathrm{~mm}$ is given by the Equation (6):

$$
\tau_{(\omega, r)}\left(R_{1}\right)=\frac{M}{2 \pi h R_{1}^{2}}
$$

A fluid is Newtonian if its dynamic viscosity is independent of stress and shear duration. There are a large number of fluids very commonly used which have a more complex flow behavior. In the case of polymer solutions when the concentration of the polymer is greater than the overlap concentration $\left(c>c^{\star}\right)$, the interactions are essentially attractive and the polymers attract each other and attach to each other [4] [13]. Tangles of molecules appear and the polymer - polymer interactions become predominant with respect to the solvent-polymer interactions and the solution becomes complex and the flow curve is non-uniform as in the case of this work. The rheology makes it possible to characterize these fluids and to deduce the hypotheses of structuration.

The expressions of the shear rate and the stress in the air gap depend strongly on the radius of the virtual cylinder $(r)$. Because of this dependence, these two quantities are measured at the same place and for a Newtonian fluid we can write: $\tau(r)=\eta \dot{\gamma}(r)$. In practice, rheometers indicate average values of stress and shear rate local in the air gap for this Newtonian fluid.

$$
\prec \sigma \succ=\frac{R_{1}^{2}+R_{2}^{2}}{4 \pi H R_{1}^{2} R_{2}^{2}} M \quad \text { and } \prec \dot{\gamma} \succ=\frac{R_{1}^{2}+R_{2}^{2}}{R_{2}^{2}-R_{1}^{2}} \omega
$$

If the fluid is not Newtonian, man can expect a difference between the law of actual behavior of the material $\sigma=f(\dot{\gamma})$ and that given by the rheometer $\prec \sigma \succ=f(\prec \dot{\gamma} \succ)$. However, when the cell used has a small difference that is to say when $R_{2}-R_{1} \prec \prec R_{1}$, it is possible to confuse the macroscopic law of the rheological behavior given by the rheometer with most non-Newtonian local rheological behaviors. In this work, it is essential to use a wide gap rheometer to identify the actual varying behavior of the sheared fluid.

\subsection{Numerical Simulations in 2D and 3D Speed Profiles}

1) Numerical Simulation in $2 D$

The second work of our rheological study after the first which allowed to establish the laws of the profiles of speed will lead to their simulation in the centered Couette geometry with a wide gap: the inner cylinder is moving at an angular velocity $\omega$ (rd./s) or controlled by $R_{e}$ (Reynolds number), the outer cylinder is fixed, for a polyethylene oxide solution with a concentration of $1.8 \mathrm{wt} \%$. According to Equation (3) and Equation (4), two profiles of dimensionless ve- 
locities are used: The first profile of Figure 1, continuous lines, is obtained from the ratio of Equation (3) relative to the effective value (i.e. $R_{1}$ maximum value of this speed $V_{0}(\mathrm{~mm} / \mathrm{s})$ at the level of the inner cylinder $\left.R_{1}\right)$. The second triangle profile is also obtained from the ratio of Equation (4), with respect to its same effective value $V_{0}(\mathrm{~mm} / \mathrm{s})$. The two velocity profiles are obtained following numerical simulations with MATLABR2008b at an angular velocity $\omega=0.9$ (rd./s) and at $R_{e}=0.0075$.

2) $3 \mathrm{D}$ numerical simulation and analysis

The main interest of 3D modeling is to see the velocity profiles in spatial mode in order to graph the different zones of the velocity profile and the conditions of inhomogeneous flows in steady state Figure 2(a). It is important to understand that the local rheological model used must obey certain conditions so that blocked regions and flowing regions can coexist, we will see further. Indeed, in this case, the same material, subjected to the same shear stress $\tau_{c}(\mathrm{~Pa})$, must be simultaneously present in two distinct states of deformation Figure 2(b). A mathematical condition that allows this coexistence is the existence of an unstable zone in the flow curve of the material [14] [15] [16], due to a decrease in the rheological law within a certain range of shear rates. But as the portion of instability is not a general case of the curves of flow; the case of foams, how is the coexistence of bands at different shear rates possible? [17].

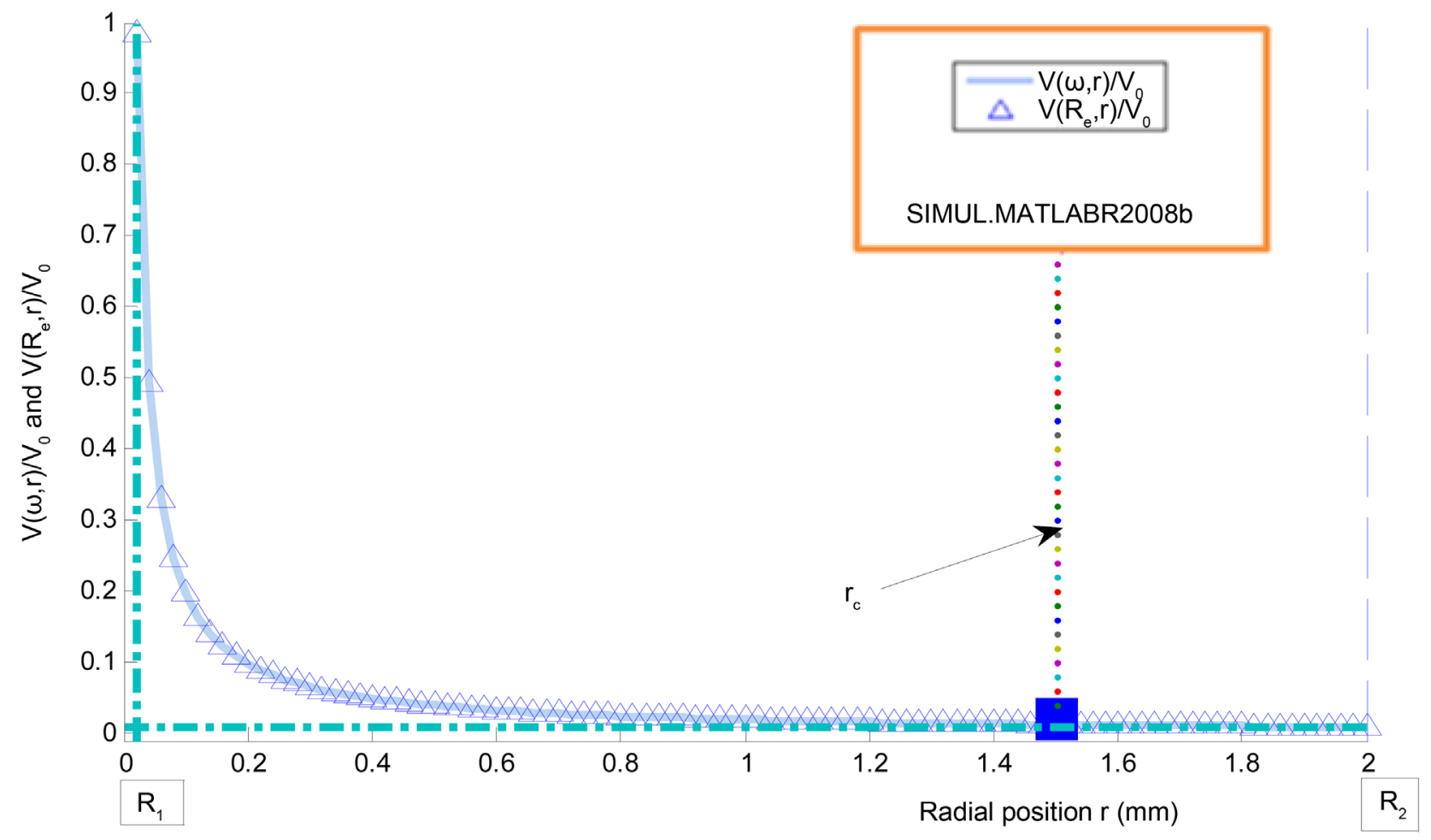

Figure 1. 2D sized air velocity profiles in the air gap of a Couette cell. (a) Modeling according to the initial theoretical law of the fluid velocity of $V(\omega, r) / V_{0}$ (continuous line); (b) According to the modified theoretical law of the fluid velocity $V\left(R_{e}, r\right) / V_{0}$ (triangular marks). $r_{c}$ marks the interface between the solid zone and the liquid zone for a flow in the geometry. The variation of the shear rate with the velocity profile from $\dot{\gamma}_{2}\left(\mathrm{~s}^{-1}\right)$ to $\dot{\gamma}_{1}\left(\mathrm{~s}^{-1}\right)$ is observed before falling into the blocked zone. 


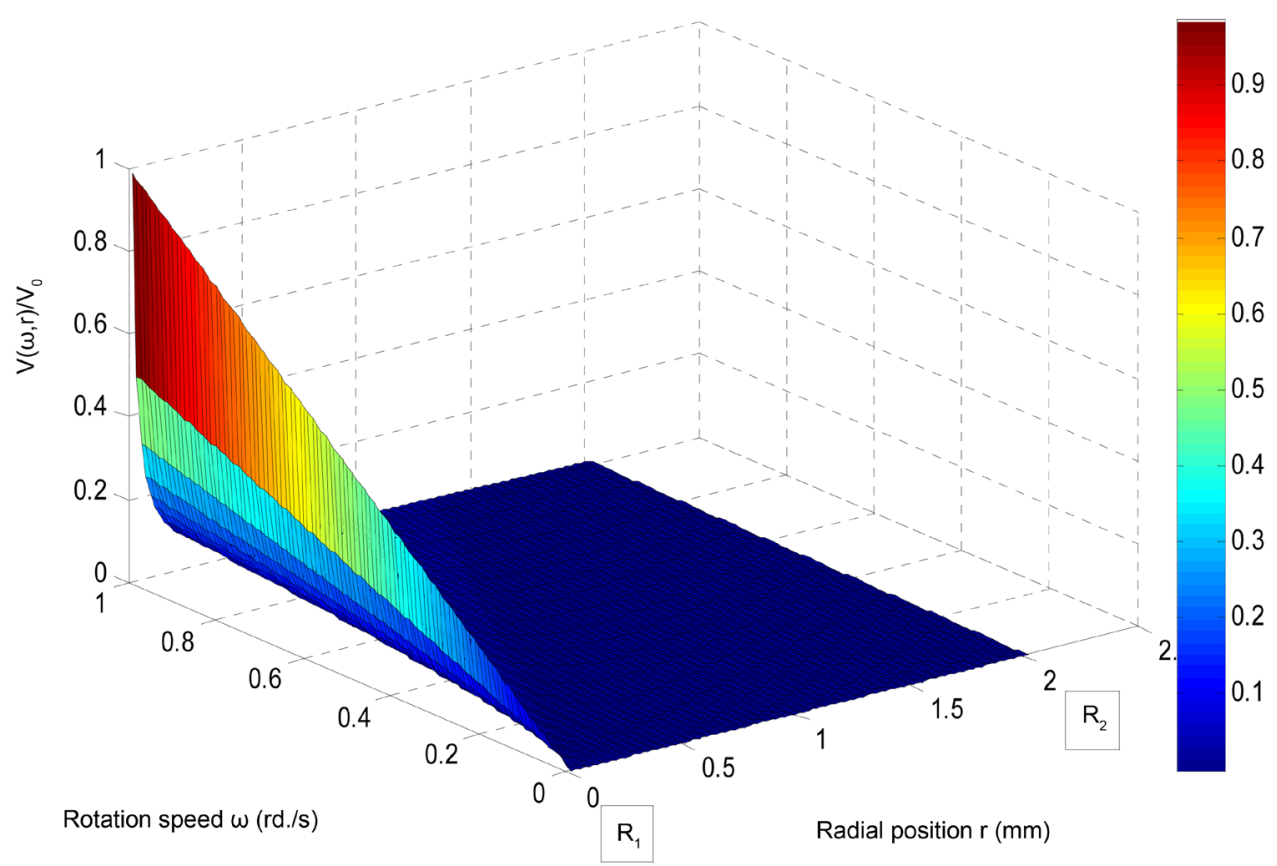

(a)

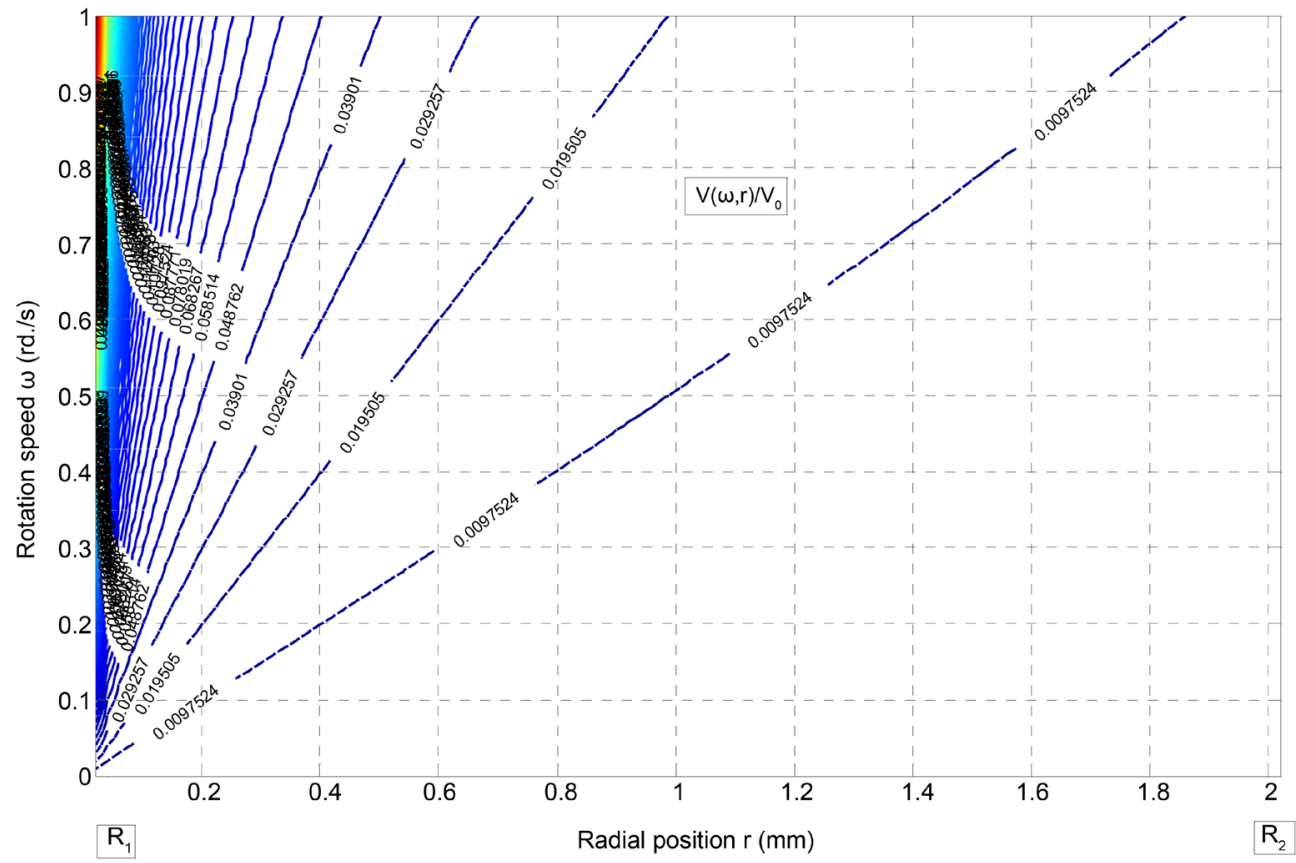

(b)

Figure 2. (a) Resilient velocity profiles $V_{(\omega, r)}(r) / V_{0}$ and $V_{\left(R_{e}, r\right)}(r) / V_{0}$ in $3 \mathrm{D}$ showing blocked regions and flowing regions. The color bar informs us about the evolution of the magnitude of the velocity profiles as a function of the parameters on which they depend in the air gap. Thus the high shear level of 0.65 to 1 at high speed in a restricted air gap develops high shear rates with very low viscosity. This viscosity increases gradually with the reduction of the shear rate from 0.65 to 0.35 and then from 0.35 to 0 with the increase of the air gap: we pass from the flow zone to the blocked zone. (b) The profiles of the same velocities in the plane $(\omega, r)$ showing a localization around the cylinder $R_{1}$ and the trajectory of the projectile of the fluid jets, the direction of rotation is that of the hands of a watch. 
Works of [4] [13] and that the fluid appears as a threshold fluid, that is to say that the applied stress must exceed a certain critical value called threshold stress for the flow to begin to occur. Many authors have specified this notion of threshold constraint [18] [19] [20] [21]. These materials may be solid under a certain level of stress, and therefore have a certain arrangement within which the elements are blocked, and liquids beyond a certain constraint, and thus have an indifferent arrangement and movable elements. These fluids undergo a smooth transition from the solid regime to the liquid regime around a given stress, which means that its flow curve $\left(\tau_{v s} \dot{\gamma}\right)$ presents, in a logarithmic representation, a plateau with low velocity gradients. Its apparent viscosity tends to infinity when $\dot{\gamma}\left(\mathrm{s}^{-1}\right) \rightarrow 0$.

3) Observations

For the two 2D curves of Figure 3(a) and Figure 3(b), a discontinuity in the slopes of the velocity profiles is observed in this Couette geometry. This situation reflects the heterogeneity of the shear rate and, also of the stresses in the fluid. The velocity profiles each have an almost constant slope $\dot{\gamma}_{2} \quad\left(\mathrm{~s}^{-1}\right)$ over a significant distance, and they fall to $\dot{\gamma}_{1}\left(\mathrm{~s}^{-1}\right)$ as in and then tend to zero and remain around this value for larger distances of the inner cylinder. They thus correspond to the velocity profiles obtained for a shear flow of giant micelles [22] in a Couette viscometer by laser velocimetry approach (PIV: Particle Image Velocimetry) and nuclear velocimetry (MRI: Magnetic Resonance Imaging), for granular materials [23], for polymer suspensions [24], and more generally for complex fluids [25] [26] [27].

This rheological study not only leads to the obtaining of rheological quantities of fluids, but also provides a window through which other complementary characteristics are deduced, particularly the behavior that binds the stresses to the flow of the fluids. Fluids according to theoretical, linear and non-linear mathematical models existing in the current literature [28]. The simulation of the two models of theoretical mathematical relation which are established in Equation (3) and Equation (4), will make it possible to understand and to identify the material by its mechanical behavior studied through the curves of the profiles of speeds from which derives the shear rate. The conditions of appearance or not of flow can be summarized as follows:

$$
\left\{\begin{array}{l}
r_{c} \prec r \prec R_{2} \Rightarrow V_{(\omega, r)}=0 \\
\text { and } \\
R_{1} \prec r \leq r_{c} \Rightarrow V_{(\omega, r)}=\frac{\omega R_{1}^{2}}{R_{2}^{2}-R_{1}^{2}}\left(-r+\frac{R_{2}^{2}}{r}\right) \text { ou } V_{\left(R_{e}, r\right)}=R_{e} K\left(-r+\frac{R_{2}^{2}}{r}\right)
\end{array}\right.
$$

4) The critical values of rotation speed and Reynolds number

In order to show all the influence of the angular velocity or of the Reynolds number on the modeling of the velocity profiles and consequently on the flow of our fluid in the Couette cylinder, we will again carry out two representations of the same profiles sized with the variation of these two parameters Figure 3(a) 


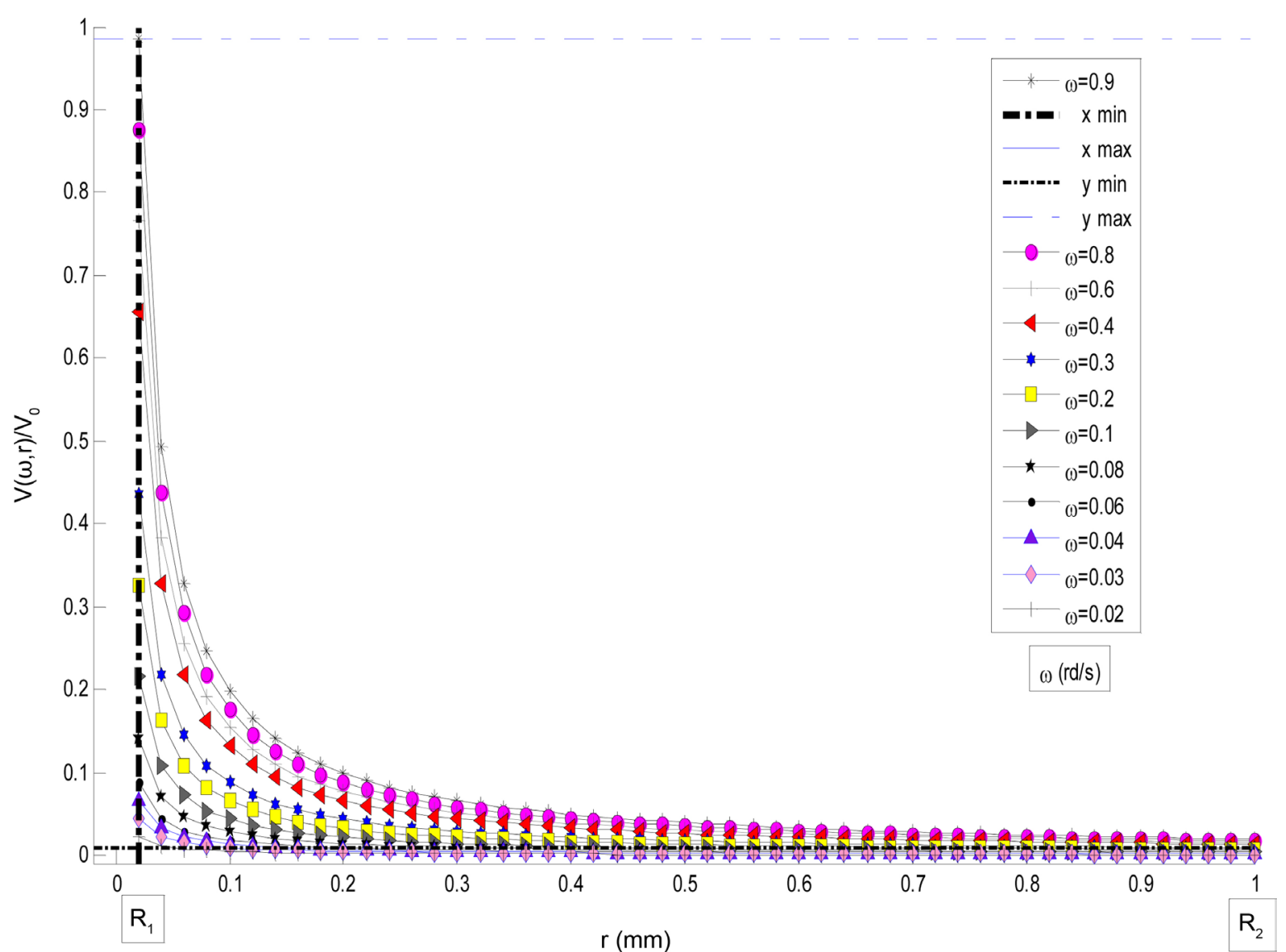

(a)

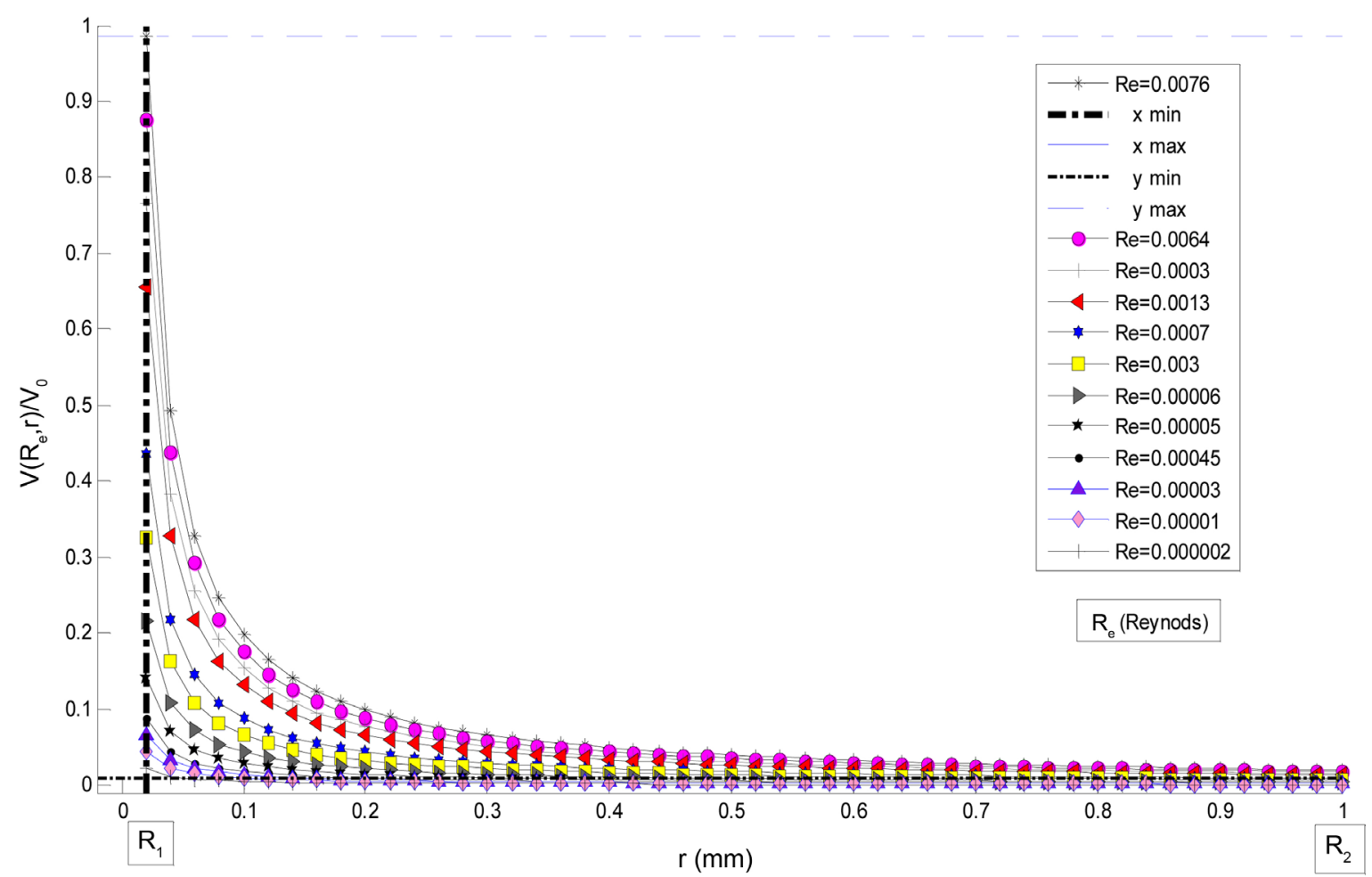

(b) 

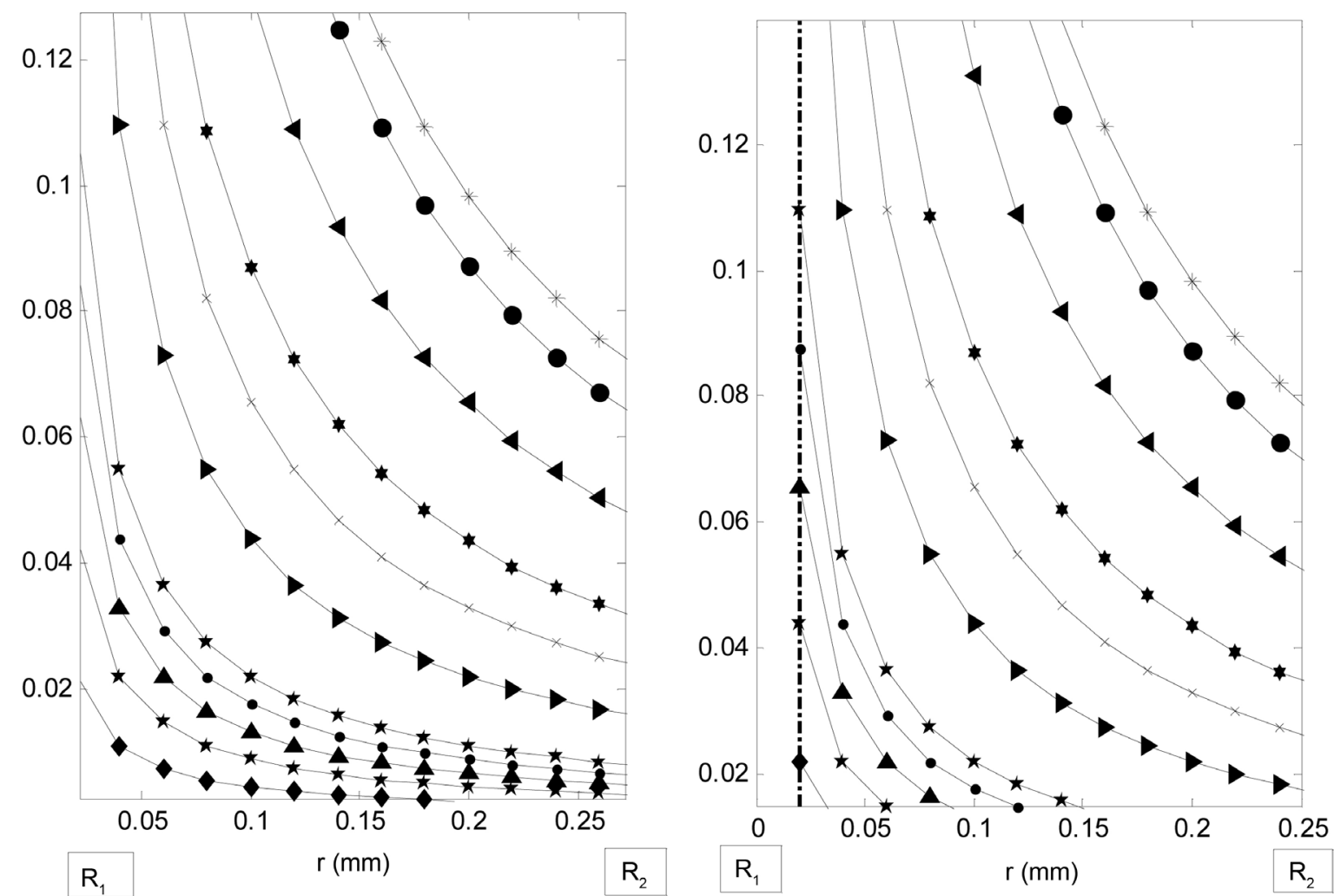

(c)

Figure 3. Profiles of local speeds $2 \mathrm{D}$ in the air gap of a Couette cell with the variation of the experimental parameters (a) $V(\omega, r) / V_{0}$ for different rotational angular velocities $(0.02$ to 0.9 (rd./s) imposed on the inner cylinder, (b) $V\left(R_{e}, r\right) / V_{0}$ for different Reynolds number (0.000002 to 0.0076), (c) zoom out to (a) and (b).

and Figure 3(b). We observe in the two cases of 2D representation of Figure 3 that for higher velocities of rotation $\omega>\omega_{c}=0.8$ (rd./s) which correspond to $\left.R_{e}>R_{e c}=54 \times 10^{-4}\right)$ the reduced velocity profiles $V_{(\omega, r)} / V_{0}$ and $V_{\left(R_{e}, r\right)} / V_{0}$ have non-zero values throughout the air gap and remain very close to one another. This is not the case for lower rotational speeds, precisely for $\omega<\omega_{c}=0.6$ (rd./s) which correspond to $R_{e}<R_{e c}=3 \times 10^{-3}$, shear is more and more localized in the vicinity of the inner cylinder with the decrease of this rotation speed or of the Reynold number and the zoom out of Figure 3(c) shows it. This also shows the results which corroborate with the results obtained in MRI in the works of [23] [24].

5) Location of the shear

When the stress field in geometry is heterogeneous, the shear rate is zero in the zones where the shear stress $\tau$ is less than the flow threshold $\tau_{c}(\mathrm{~Pa})$. This is the case in cylindrical pipe flows where the shear stress is proportional to the radius: the material is sheared only near the walls and the central part of the material (where $\tau<\tau_{c}$ ) is transported at constant speed, then the shear is localized. This problem is found in the rheometry of threshold fluids where the velocity field measured locally by MRI during the flow of a concentrated emulsion 
in the Couette geometry is represented. In the case of our study by a numerical simulation in Figures 1-3, we note that the material is not sheared in the whole of the air gap: there is a zone near the outer cylinder where the speed is zero. Moreover, the size of the sheared zone decreases when the rotation speed $\omega$ of the inner cylinder or the Reynolds number $R_{e}$ is decreased as shown by the work [23] [24] [29].

Thus, we can say that at low rotational speeds which correspond to very low numbers of Reynolds Figure 3(a) and Figure 3(b) or (i.e. $\omega_{c}=0.02(\mathrm{rd} . / \mathrm{s}$ ) which correspond to $R_{e}=2 \times 10^{-6}$ ), the velocity profiles only deploy over a small part of the air gap close to the inner cylinder of radius $R_{1}$ therefore the shearing decreases with the decrease of the angular velocity or the number of Reynolds: there is localization of the shear, only a small fraction of the material is sheared. However, by increasing the rotational speed, we observe that the higher the rotational speed, the larger the shear fraction of the material, the greater the air gap [23] [24] [29]. The assembly then thickens and the engines can no longer rotate the inner cylinder: the system is blocked systematically. This blocking can be attributed to the rheo-thickening by the abrupt increase of the torque measured on the axis of the rheometer [24]. In order to see explicitly what has just been said in the paragraph above, a zoom out is carried out in Figure 3(a) and Figure 3(b). Moreover, the discontinuity of the shear rate in two regions shows the existence of at least two shear rates: $\dot{\gamma}_{2}$ is constant for the liquid zone and $\dot{\gamma}_{1} \rightarrow 0$ for the solid zone in Figure 4. This behavior when varied $\dot{\gamma}, \dot{\gamma}_{1}<\dot{\gamma}<\dot{\gamma}_{2}$ is closer to what is usually described as flow in shear bands, i.e. the flow is heterogeneous (nonlinearity of the velocity gradient with several regions sheared locally at different shear rates [30] [31] [32] [33]. It may thus appear paradoxical that the same material, subjected to a uniform or substantially heterogeneous shear stress $\tau_{c}(\mathrm{~Pa})$ between two coaxial cylinders or between two plates, may be simultaneously present in two distinct states in different regions of the flow. This situation is explained mechanically by a fluctuation of the stress, which translates into another term by the relation of dependence between stress and shear rate.

6) Shear band

The fact that we obtain two different zones at different shear rates $\dot{\gamma}<\dot{\gamma}_{2}$ and $\dot{\gamma}>\dot{\gamma}_{1}$ translates a mechanical instability (existence of an elastic limit) [34] as in the case of micelles giant. It is expected that a phase coexistence for $\dot{\gamma}_{1}<\dot{\gamma}<\dot{\gamma}_{2}$ in the form of a heterogeneous flow consisting of two locally sheared regions with characteristic shear rates $\dot{\gamma}_{1}\left(s^{-1}\right)$ and $\dot{\gamma}_{2}\left(s^{-1}\right)$ Figure 4. Such a so-called shear bend flow was clearly demonstrated for the first time in cylindrical Taylor-Couette geometry a decade ago thanks to the use of velocimetry techniques locally probing the velocity field at $d$ rheology experiments [30]. The shear bands are frequently accompanied by large fluctuations of the velocity field [31]. The team of Sandra Lerouge was able to explain these fluctuations as emanating from an elastic instability in the "aligned phase" [35]. While the inertial 


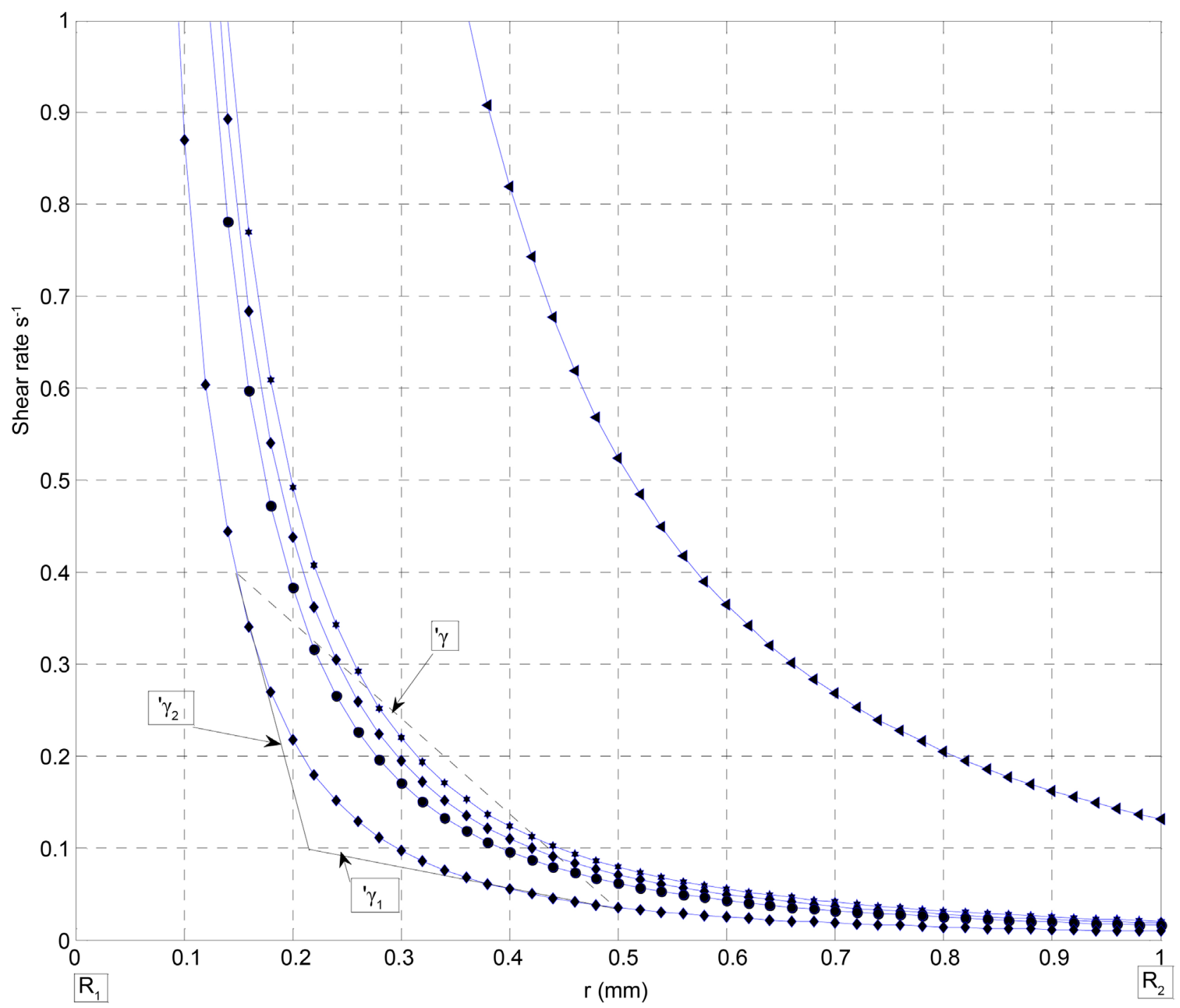

Figure 4. Evolution of the shear rate for the local velocity profiles in the air gap of a Couette cell with the variation of the experimental parameters. The variation of the shear rate with the $\dot{\gamma}_{2}\left(\mathrm{~s}^{-1}\right)$ flow velocity profile at $\dot{\gamma}_{1}\left(\mathrm{~s}^{-1}\right)$ is observed before falling into the blocked zone (dead zone).

force (centrifugal force) is the driving force of Taylor-Couette instability in a simple fluid, elastic in stability is generated by the (centripetal) forces exerted along curved current lines [33]. In the case of flow in shear bands, instability develops only in the heavily sheared region. There follows a very particular flow in which the Taylor-Couette rollers deform the interface between the two shear bands. Mechanically, these fluids can be considered as flow stress fluids in the sense that they can- not flow at steady state unless the stress applied to them exceeds a value critical and finite. This property results from the existence of a continuous network of interactions (i.e. blocked structure) between the elements, which must be broken for flow to occur. The elastic limit of the material is therefore related to the resistance of this network. On the other hand, on our curve in Figure 4 a discontinuity in the slope of the velocity profile measured 
during flows for very low speeds.

The velocity profile at an almost constant slope at a distance and sharply decreases to near zero and maintains this value for greater distances. We still have the coexistence of a solid region and a liquid region in such a flow. However, there is here a discontinuity of the shear rate at the interface between the two regions: the shear rate is critical at a point of shear rate $\dot{\gamma}_{c}\left(\mathrm{~s}^{-1}\right)$ in the liquid region and equal to zero in the solid region. This behavior is closer to what is usually called the shear band [24] [29].

The velocity profile at an almost constant slope at a distance and sharply decreases to near zero and maintains this value for greater distances. We still have the coexistence of a solid region and a liquid region in such a flow. However, there is here a discontinuity of the shear rate at the interface between the two regions: the shear rate is critical at a point of shear rate $\dot{\gamma}_{c}\left(\mathrm{~s}^{-1}\right)$ in the liquid region and equal to zero in the solid region. This behavior is closer to what is usually called the shear band [24] [29].

7) Position of location and link between microscopic and macroscopic measurements

Since the paragraph 3.2, the explanatory approach of the behavior of our material in the geometry to start and will continue for the determination of the quantities that will allow to establish the law of complete behavior of the material. It is necessary to determine the threshold of the material which will be added to a power law. In fact, the position of location shows in the Couette geometry the area or shear fluid in fraction and the zone or the fluid shears totally according to thicknesses $r_{c}(\mathrm{~mm})$ Figure 5 with the increase of the angular velocity $\omega$ (rd./s) or the Reynold number $R_{e}$. This value of $r_{c}(\mathrm{~mm})$ is a geometric factor which makes it possible to obtain, at first glance, the apparent shear rate $\dot{\gamma}_{c} r_{c}$ imposed in the sheared region $r_{c}(\mathrm{~mm})$. Then, as the behavior of Figure 1 and Figure 2(a) shows the inhomogeneity of the stress in the material studied, the variation in the state of the liquid structure in solid, while the shear stress $\tau(R)$ at a radial position $(r)$ in the centered duvet geometry is written [36]:

$$
\tau(R)=M / 2 \pi h R^{2}
$$

$M$ is the torque applied to the inner cylinder of radius $R_{1}$ and of height $\mathrm{h}$ given by Equation (9) above. The measurement of the critical radius $r_{c}(\mathrm{~mm})$. To which the material ceases to flow imposes knowledge of the critical stress characterizing the flow threshold by other means and the knowledge of the torque applied to the inner cylinder will make it possible to deduce the location $r_{c}$ (mm) as in Equation (9) (i.e. $\tau_{c}\left(r_{c}\right)=M / 2 \pi h r_{c}^{2}$ ).

We obtain a threshold stress $\tau_{c}(\mathrm{~Pa})$, a constraint which is sometimes too low to be detected by a simple experiment such as an inclined plane test [37]. Thus, although the solution does not seem to behave macroscopically like a threshold fluid, it sometimes has a very low threshold. It is also noted that there exists a shear rate associated with this flow threshold given by Equation (10) below in a Couette cell. 


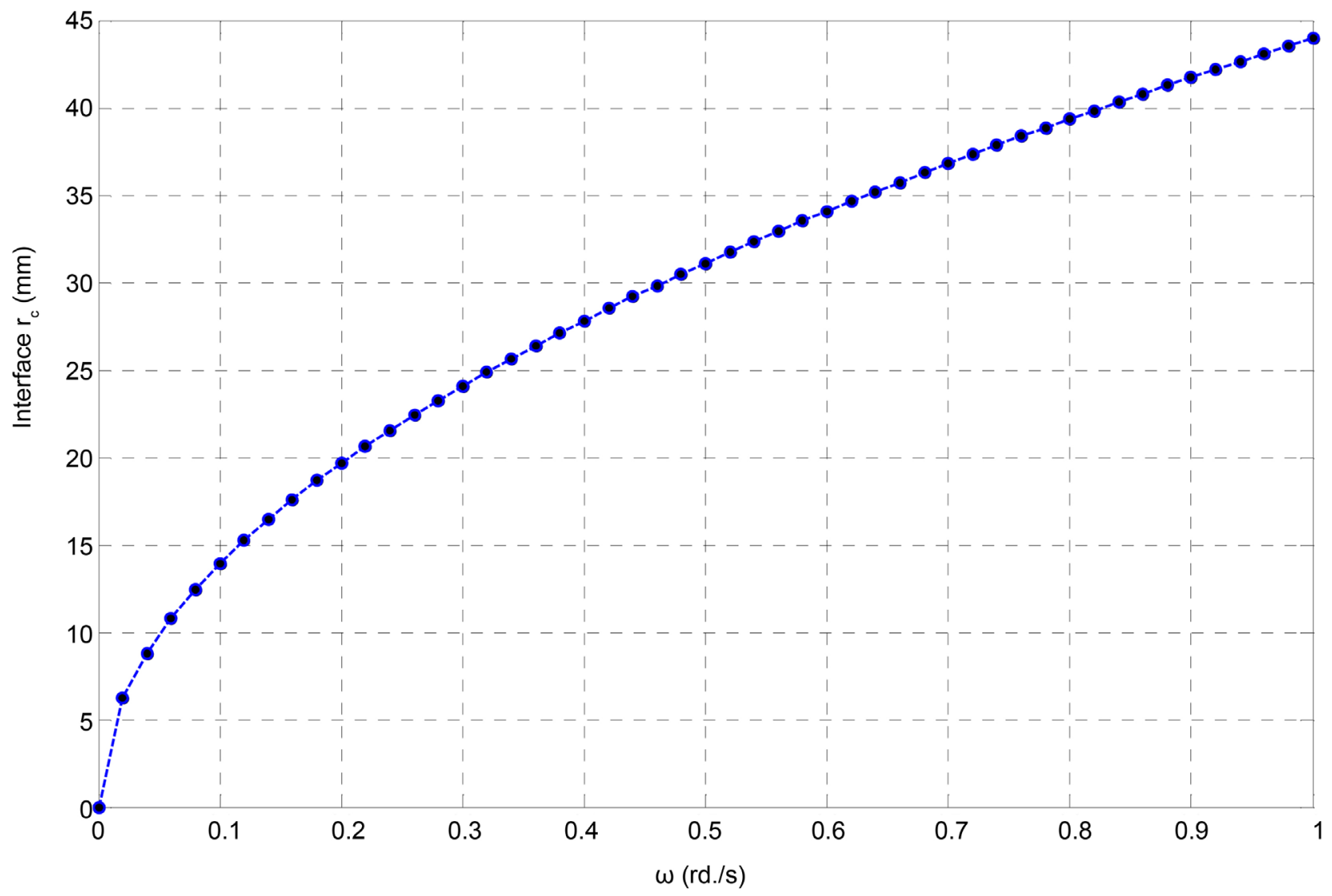

Figure 5. Evolution of the size of the interface $r_{c}$ between the sheared zone and the unsheared zone as a function of the speed of rotation for a large gap $4.5(\mathrm{~cm})$. When the air-gap is reduced by $2(\mathrm{~mm})$, the interface becomes linear.

$$
\dot{\gamma}(r)=\frac{\partial V(r)}{\partial r}-\frac{V(r)}{r}
$$

At the interface of the sheared and non-sheared zones characterizing the stoppage of flow, the associated shear rate is given by the slope of the velocity profiles. This rate corresponds to $\dot{\gamma}_{c}\left(\mathrm{~s}^{-1}\right)$, and below this threshold $\dot{\gamma}_{c}\left(\mathrm{~s}^{-1}\right)$, value, there is no stable flow. The existence of a threshold shear rate associated with a stress threshold is a general property of threshold materials discovered recently [36]. Thus, for given $\dot{\gamma}_{c}\left(\mathrm{~s}^{-1}\right)$, to lower values, the whole sample is sheared. Also for higher values $\dot{\gamma}_{c}\left(\mathrm{~s}^{-1}\right), r_{c}(\mathrm{~mm})$ increases and the fluid is sheared throughout the air gap. $\dot{\gamma}_{c}\left(\mathrm{~s}^{-1}\right)$ is the same for two different air gaps [23]. Also in the gap, the local shear rate decreases exponentially in Figure 4 and Equation (5). This leads to the variation of the constraint in $1 / r^{2}$ as we will show by the conservation of the moment. The viscosity also varies according to the equation in the air gap, and therefore two questions arise: can one define a viscosity for the viscous solution which could completely characterize its resistance to flow? Will it have compatibility for a viscous solution between the "overall" viscosity measured macroscopically without worrying about the speed profile and the "local" viscosity of the solution? [23]. These two questions thus pose problems between macroscopic and microscopic measurements. And ac- 
cording to the work of Nicolas Huang [30]: It is possible to measure the viscosity of the material from a profile locally, which is to say at a given distance, $r(\mathrm{~mm})$ from the axis of the cylinder of speeds. If it is possible to define a viscosity to the material, then, for the same stress, the local viscosity resulting from a velocity profile must be identical to the overall viscosity measured in the classic Couette cell experiments. Conservation of the moment in $r(\mathrm{~mm})$ and in $R_{1}$ leads to the determination of the local stress which varies as a function of $1 / r^{2}$.

$$
\sigma(r)=\sigma_{i} \frac{R_{i}^{2}}{r^{2}}
$$

Since the local shear rate is the spatial derivative of the velocity profile (slope of the profile), the local viscosity can be calculated as compared to the overall viscosity. The localization of shear is due to the fact that the shear stress $\tau_{(\omega, R)}$ measured at low rotational velocity $\omega(\mathrm{rd} . / \mathrm{s})$ of the inner cylinder passes below the threshold at the interface and of Equation (12), the interface of the two zones of flow and of non-flow (dead zone) by the equation.

$$
R_{c}(\omega)=R_{1} \sqrt{\frac{\tau(\omega, r)}{\tau_{c}}}
$$

When $\omega(\mathrm{rd} . / \mathrm{s})$ tends to $0, R_{c}(\omega)$ then tends to $R_{1}$, i.e. the thickness of flowing material tends to zero and the shear stress $\tau_{\left(R_{1}, \omega\right)}$ on the inner cylinder tends towards $\tau_{c}(\mathrm{~Pa})$ [29]. The two curves in Figure 1 each have a constant slope over a significant length of the air gap up to the value $r_{c}(\mathrm{~mm})$, and then the two slopes suddenly fall to a value which tends to zero for the large values of $r(\mathrm{~mm})$ on the rest of the gap. This value point $r_{c}(\mathrm{~mm})$ of the Equation (12) of the Couette geometry is defined in several ways [38] [39]. It is preferred to define it here as the position separating the discharge region from the rigid, or equivalently as the limit value of the radius at which the deformation rate is zero. This shows that we are in the presence of the coexistence of two regions, one solid therefore without flow or not sheared, and the other liquid sheared in such and such a fluid Figures 6(a)-(c). All observations and analyzes show that the velocity profile in the air gap is non-Newtonian, but it is possible to obtain a velocity profile relationship as before by postulating a constituitve law for the fluid. Thus, assuming that the fluid obeys a power law of Ostwald of the Equation (13):

$$
\sigma=k \dot{\gamma}^{n}
$$

8) Medialization

This situation is presented as the behavior of stress fluids in simple shear which has a viscosity plateau. It is modeled according to Equation (14).

$$
\left\{\begin{array}{l}
\tau \prec \tau_{c} \Rightarrow \dot{\gamma}=0 \\
\text { but: } \\
\tau \geq \tau_{c} \Rightarrow \tau=f(\dot{\gamma})=k \dot{\gamma}^{n}
\end{array}\right.
$$

and in this case, the final law of the behavior is:

$$
\tau=\tau_{c}+K \dot{\gamma}^{n}
$$




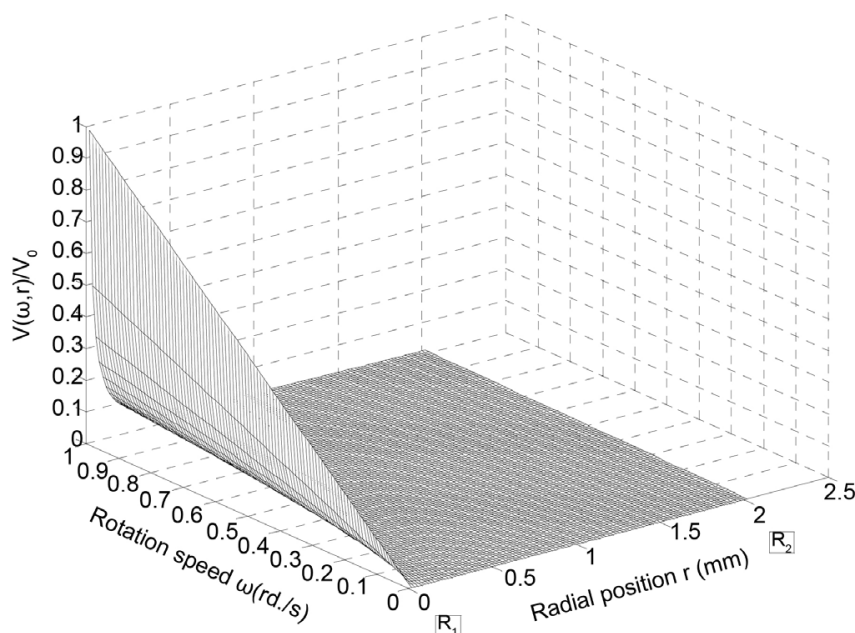

(a)

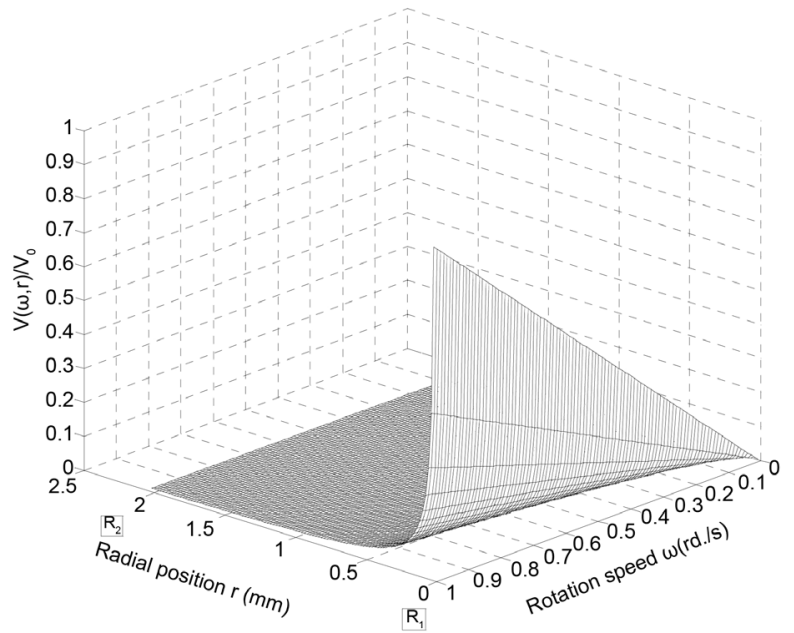

(b)

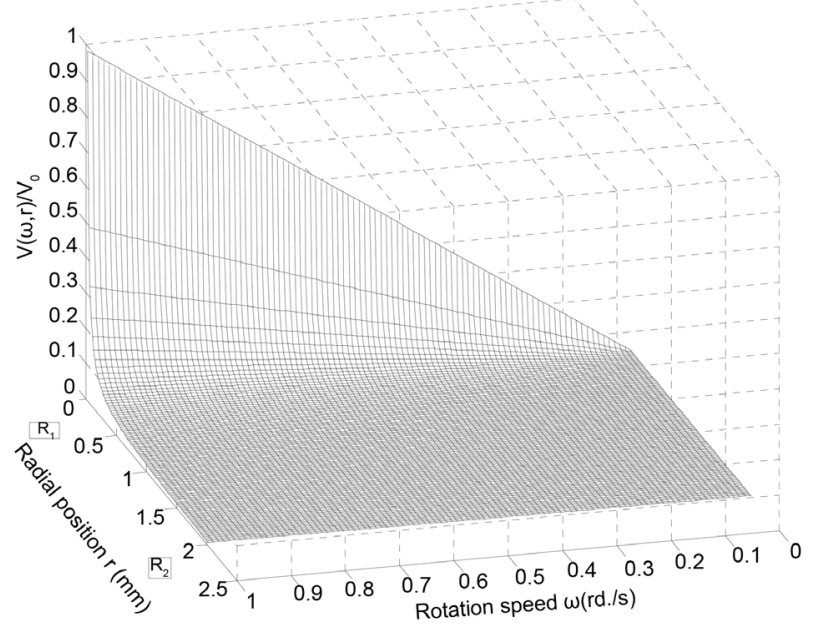

(c)

Figure 6. (a) Façade of the velocity profiles in the air gap of a Couette cell (dominance: partially sheared zone), and possibilities of encountering the flowing zone and the blocked zone from the parameters $(\omega, r)$. The level of the blocked band varies from $\omega=$ 0.002 to 0.9 (rd./s) when the sheared space varies from $r=0.002$ to $2(\mathrm{~mm})$. The locking is very important over a large space of the air gap $(r)$, moving away from the cylinder $R_{1}$ progressively with the decrease in the rotational speed or in the number of Reynolds. (b) Behind plane facade of the velocity profiles in the air gap of a Couette cell. The velocity profile (dominance: totally sheared zone) shows the dominance of the sheared zone on the blocked zone with the increase of the two parameters always in the same plane $(\omega, r)$. The interface $\left(r_{c}\right)$ of the last profile is the first point of the border of this profile when it appears parallel to the axis $r . r_{c}=1.5(\mathrm{~mm})$ and this value increases with the decrease of the angular velocity or the Reynolds number. (c) The basic plane showing the evolution of the velocity profiles from the clear zone to the dark blocked area.

where $\tau_{c}(\mathrm{~Pa})$ corresponds to the threshold constraint, it is deduced from the equation rheological equation. The rheograms of the experimental data are plotted in the log-log coordinates. The number $n$ represents the slope of the line obtained and is also the index of structure. For $0<n<1$ the fluid is rheo-fluidifier, then $n=1$ is the Bingham case and $n>1$ the rheo-thickening case. $K$ is the consistency in $\left[\mathrm{N} \sec ^{2} / \mathrm{m}^{2}\right]$, is given by the point of intersection of the line with the axis corresponding to $\dot{\gamma}=1\left(\mathrm{~s}^{-1}\right)$. Since $\dot{\gamma}$ in cylindrical coordinates is written: 


$$
\dot{\gamma}=r \frac{\partial}{\partial r}\left(\frac{V_{(\theta, r)}}{r}\right)
$$

We have:

$$
V_{(\theta, r)}=r \omega\left(\frac{\left(R_{2} / r\right)^{2 / n}-1}{\left(R_{2} / R_{1}\right)^{2 / n}-1}\right)
$$

The system of Equation (8) is repeated for a complex fluid in the form:

$$
\left\{\begin{array}{l}
r_{c} \leq r \leq R_{2} \Rightarrow V_{(\theta, r)}=0 \\
\text { but: } \\
R_{1} \leq r \leq r_{c} \Rightarrow V_{(\theta, r)}=r \omega \frac{\left(R_{2} / r\right)^{2 / n}-1}{\left(R_{2} / R_{1}\right)^{2 / n}-1}
\end{array}\right.
$$

This model of velocity profile fairly well represents the flow of PEO solution Figure 7 and Figure 8. However, in addition to the shear rate which is not always constant Figure 9, it has a remarkable failure to take into account the localization observed during the flow of the solution at low rotation speeds, existence of a threshold is not predicted by this model. Indeed, the localization of the flow in Couette geometry is closely related to the flow threshold. Thus, in order to describe the localization, a truncated power model was proposed by Equation (20) [38] [39] [40].

$$
\left\{\begin{array}{l}
\tau \prec \tau_{c} \Rightarrow \dot{\gamma}=0 \\
\tau \geq \tau_{c} \Rightarrow \frac{\tau}{\tau_{c}}=\left(\frac{\dot{\gamma}}{\dot{\gamma}_{c}}\right)^{n}
\end{array}\right.
$$

$\tau_{c}$ and $\dot{\gamma}_{c}$ correspond respectively to the stress and the shear rate at the interface between the sheared zone and the non-sheared zone of the Equation (13). When $r_{c}=R_{2}(\mathrm{~mm})$, radius of the outer cylinder, there is no localization and the velocity profile remains analogous to that obtained by the Ostwald model.

In this case the Ostwald law is not sufficient to analyze the non-localized velocity profiles. On the other hand, in the case of low rotational speeds where the flow is localized, the characterization of the flow is performed by the truncated power law. In this case, the speed profile is given by:

$$
\left\{\begin{array}{l}
r_{c} \leq r \leq R_{2} \Rightarrow V_{(\theta, r)}=0 \\
R_{1} \leq r \leq r_{c} \Rightarrow V_{(\theta, r)}=\frac{n}{2} r \dot{\gamma}_{c}\left(\left(\frac{r_{c}}{2}\right)^{2 / n}-1\right)
\end{array}\right.
$$

A second fundamental reason for the inadequacy of Ostwald Waele's power law is that it has a linear range of viscosity at low shear rate on the log-log diagram when this law is expressed in terms of viscosity. However, the fluid is rheo-fluidifying, and therefore characterized by the decrease of the apparent viscosity when the shear rate increases. This assumes that this Ostwald power law is valid only within a certain range of shear rates. The Tile model satisfactorily represents the evolution of the viscosity as a function of the shear rate. Using the 


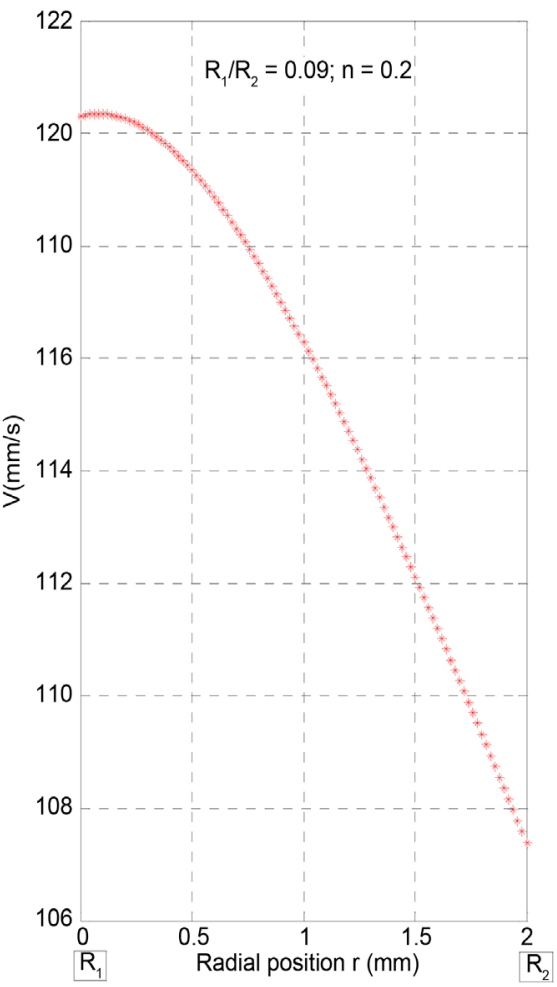

(a)

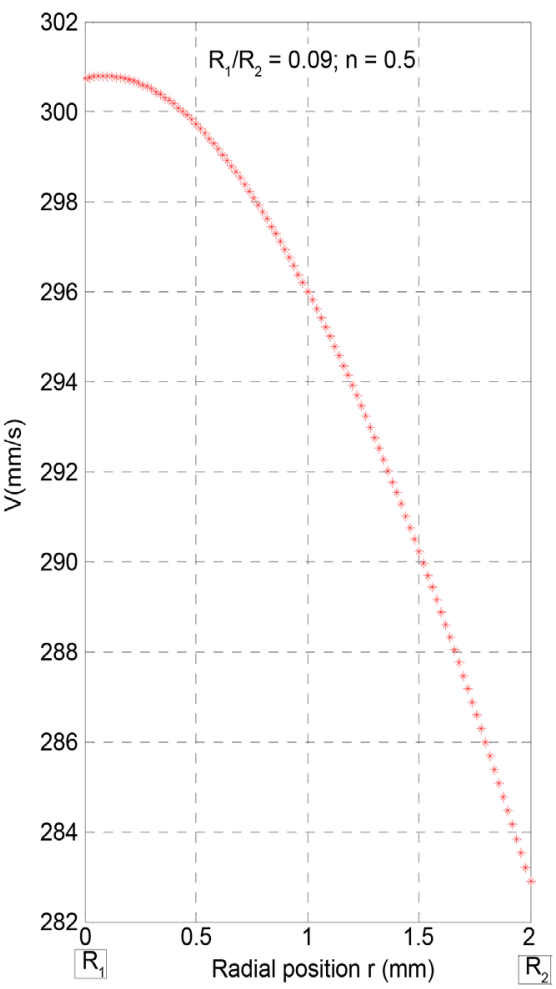

(b)

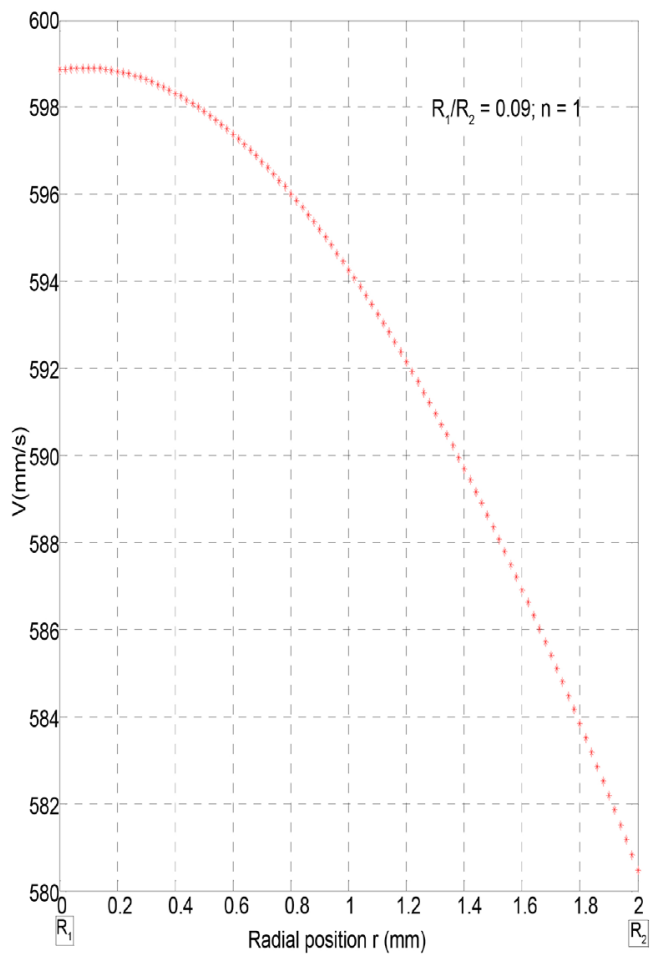

(c)

Figure 7. Velocity profiles according to the Ostwald power law for the rheo-fluidifying material in an air gap $R_{1} / R_{2}=0.99$ : (a) $n=0.2$; (b) $n=0.5$ for the rheo-fluidifying fluids and (c) $n=1$ for the Bingham fluid. In all three cases, the material presents a plateau of velocity profiles before varying linearly in the radial space up to $R_{2}$. 

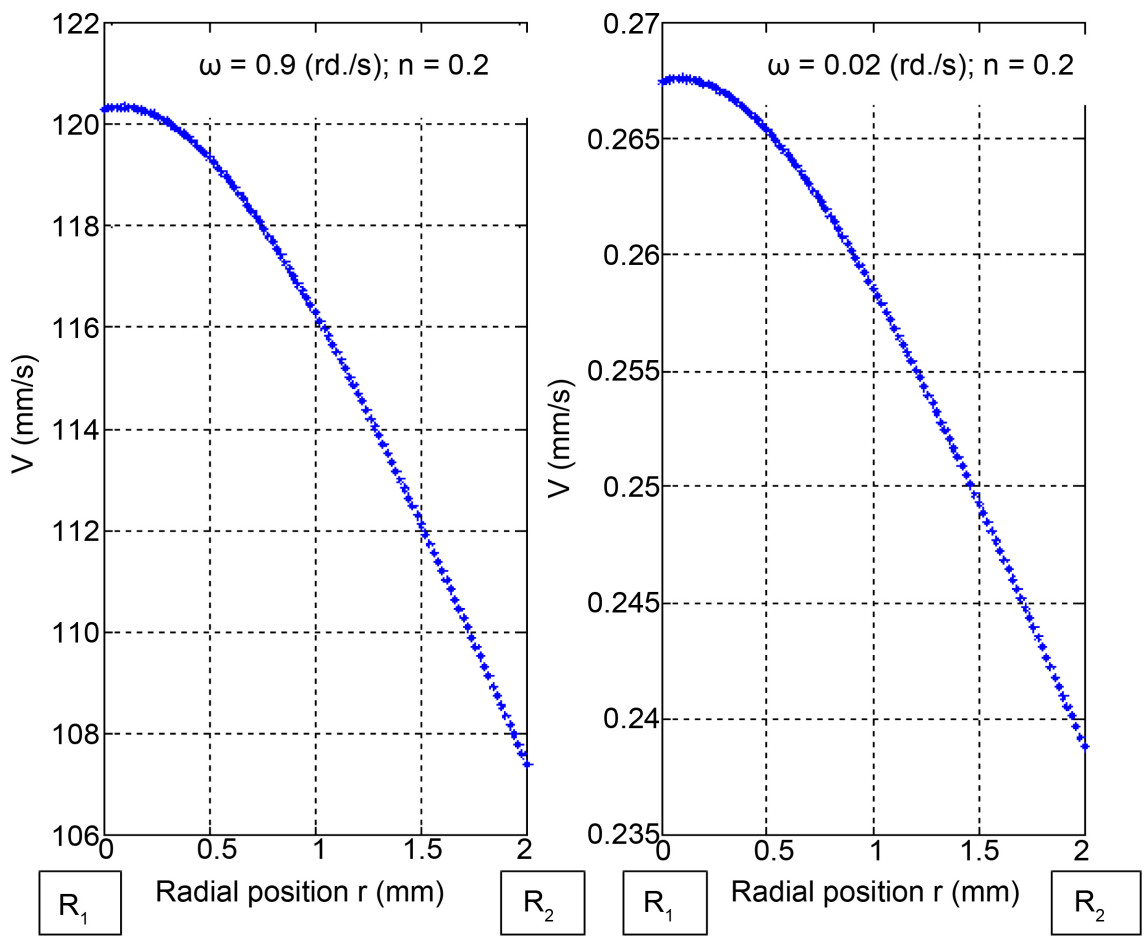

Figure 8. Velocity profiles according to the Ostwald power law for the rheo-fluidifying material in an air gap $R_{1} / R_{2}=0.99: n=0.2$. (a) Fully sheared fluid $\omega=0.9(\mathrm{rd} / \mathrm{s})$; (b) Partially sheared fluid $\omega=0.02(\mathrm{rd} / \mathrm{s})$.

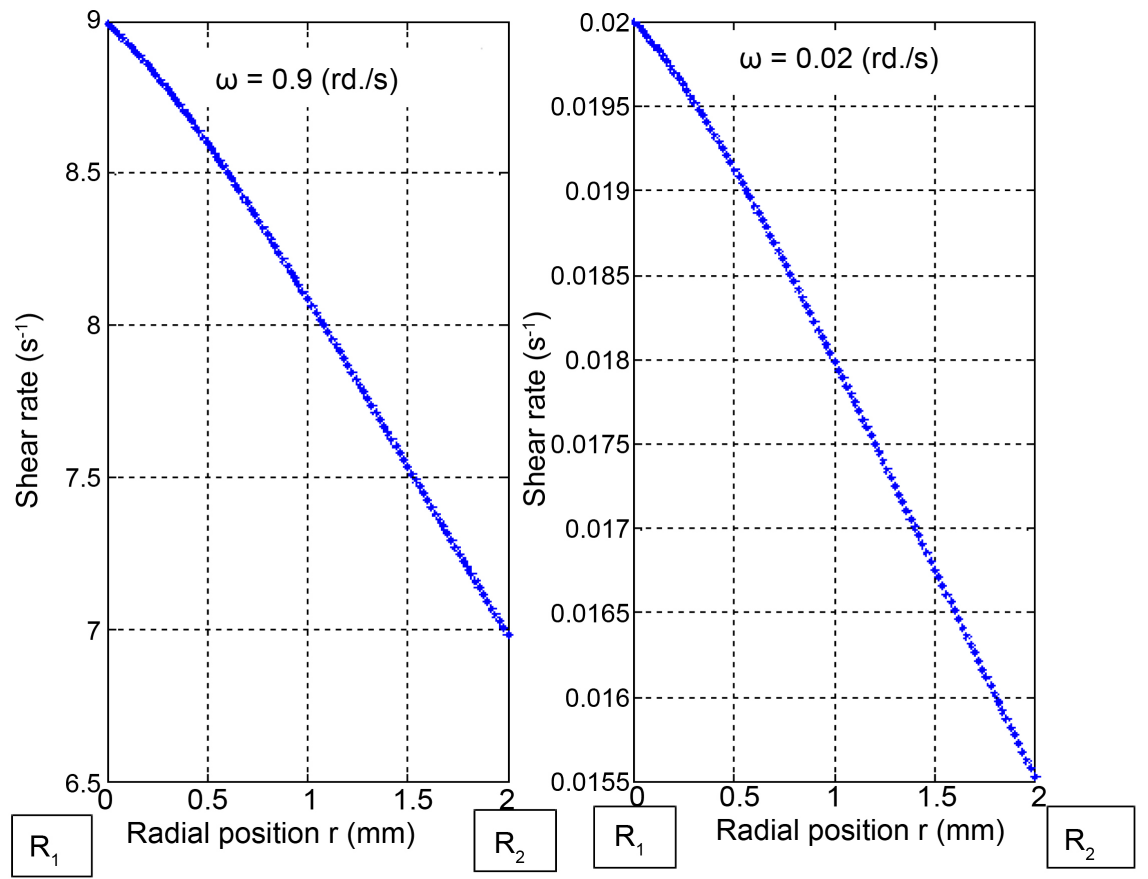

Figure 9. Ostwald power law shear rate curves for $R_{1} / R_{2}=0.99$. (a) Totally sheared fluid $\omega=$ 0.9 (rd/s); (b) Partially sheared fluid $\omega=0.02(\mathrm{rd} / \mathrm{s})$.

values of $n, r_{c}, \dot{\gamma}_{c}$ in the equation of the truncated model, we obtain the linear curves of Figure 10 as for Newtonian fluids [23]. 


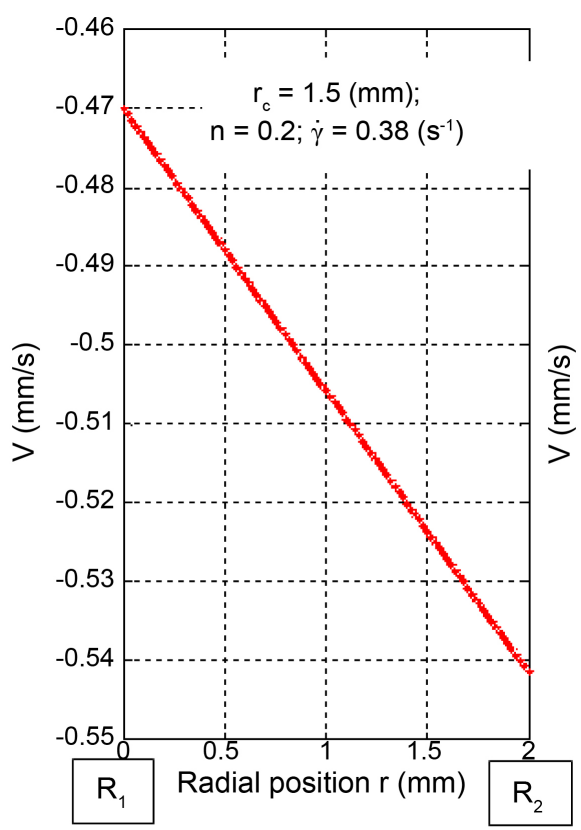

(a)

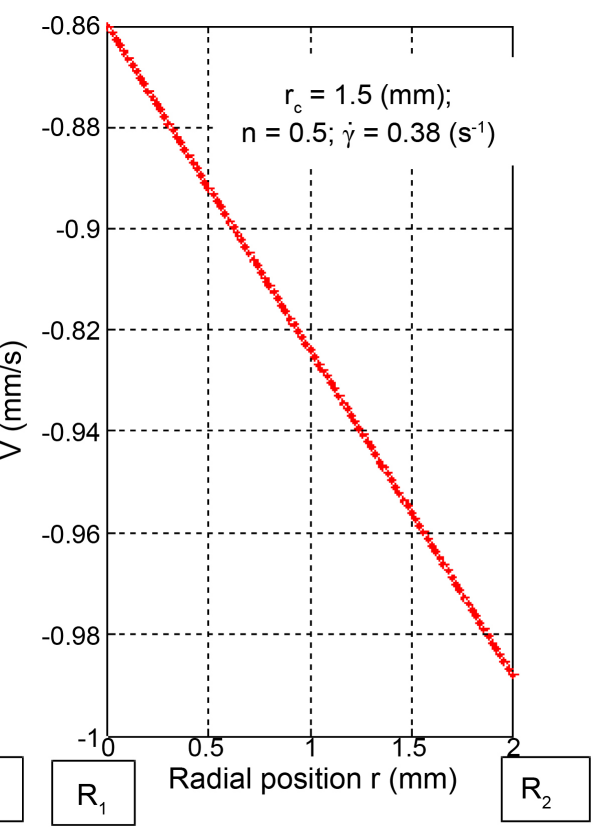

(b)

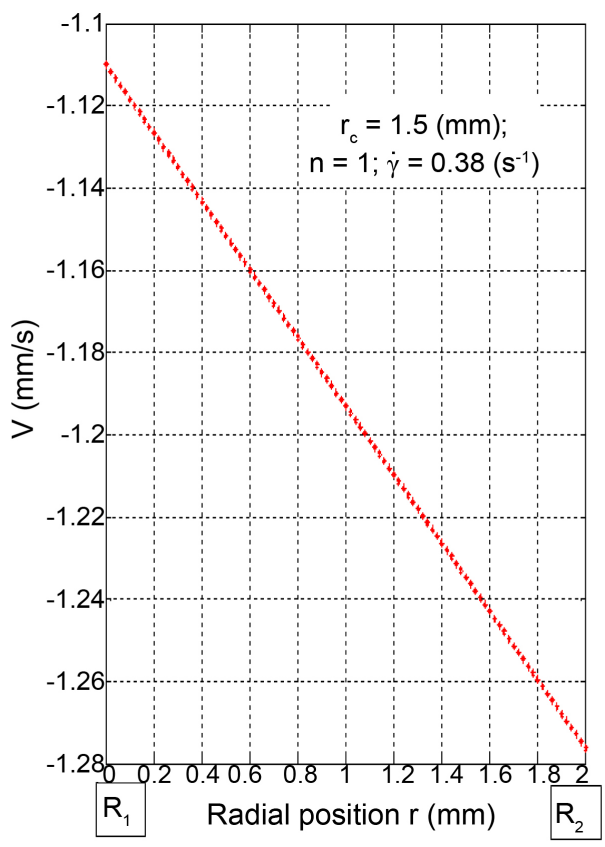

(c)

Figure 10. Velocity profiles according to the truncated power law for the rheo-fluidifying material in an air gap $R_{1} / R_{2}=0.99$. (a) $r_{c}=1.5(\mathrm{~mm}) ; n=0.2 ; \dot{\gamma}=0.38\left(\mathrm{~s}^{-1}\right)$; (b) $r_{c}=1.5$ $(\mathrm{mm}) ; n=0.5 ; \quad \dot{\gamma}=0.38 \quad\left(\mathrm{~s}^{-1}\right) ;$ (c) $r_{c}=1.5(\mathrm{~mm}) ; n=1 ; \dot{\gamma}=0.38 \quad\left(\mathrm{~s}^{-1}\right)$. In all three cases, the material has a Newtonian velocity profile. The dimensionless values of the speeds are counted positively.

\section{Conclusion}

In this paper, we have presented the basic method for identifying the flow curve of a shear fluid in a Coutte cell and then the searching for the most appropriate 
procedure for the determination of the rheological quantities that relate to this fluid. This method is simple and efficient through the results obtained numerically, but one can get rid of certain limitations related to the size of the air gap [41] which causes a flow not the identity of the material related to its properties, a priori choice of a law of behavior, flow regime in the air gap, and preliminary assessment of the flow threshold. Thus, the characterization of the polyethylene oxide solution was made possible by converting the macroscopic data (angular velocity and Reynolds number) into rheological data (behavior law), based on an analysis of the numerical results of velocity profiles obtained in the Couette geometry. The velocity profiles obtained show that the flow is a non-Newtonian behavior. There is non-zero shear throughout the air gap for higher frequency values $\left(\omega \geq \omega_{c}=0.8\right.$ radian/s $)$, corresponding to the higher Reynolds numbers $\left(R_{e} \geq R_{e c}=54 \times 10^{-4}\right)$ and localized shear around the inner cylinder for lower values of $\omega<\omega_{c}=0.6 \mathrm{rd} . / \mathrm{s}$, corresponding to the lower Reynolds numbers $R_{e}<R_{e c}=3 \times 10^{-3}$. This situation causes a shear band, hence a variation in shear rate and viscosity. This shows that the suitable model for the polyethylene oxide solution is the one which depends on the concentration and the shear rate, but in all cases, it remains as a threshold rheo-fluidifier-fluid.

\section{References}

[1] Koblan, W.E., Benchebane, A., Bekkour, K. and Allgaier, A. (2007) Rheology of Solutions of Polyethylene Oxide (PEO) at Different Molecular Weights. 42nd Annual Symposium of the French Group of Rheology-Rheology of Evolutionary Systems, Clermont-Ferrand, Janvier 2007, 361-367.

[2] Ortiz, M., De Kee, D. and Carreau, P.J. (1994) Rheology of Concentrated Poly(ethylene oxide) Solutions. Journal of Rheology, 38, 519-539. https://doi.org/10.1122/1.550472

[3] Rossi, S., Luckham, P.F., Zhu, S., Briscoe, B.J. and Tadros, T.F. (1997) Influence on Low Molecular Weight Polymers in the Rheology of Bentonite Suspensions. Oil \& Gas Science and Technology, 52, 199-206. https://doi.org/10.2516/ogst:1997019

[4] Koblan, W.E., Benchebane, A., Bekkour, K. and Allgaier, A. (2009) Using the Cross Model for the Rheological Characterization of Polymer Solutions. 44th Annual Colloquium of the French Group of Rheology, Strasbourg, 4-6 November 2009, 261-264.

[5] Gauri, V. and Koelling, K.W. (1997) Extentional Rheology of Concentrated Poly(ethylene). Rheologica Acta, 36, 555-567. https://doi.org/10.1007/BF00368133

[6] Kalashnikov, V.N. (1994) Shear-rate Dependent Viscosity of Dilute Polymer Solutions. Journal of Rheology, 38, 1385-1403. https://doi.org/10.1122/1.550550

[7] Powell, R.L. and Schwarz, W.H.J. (1979) Nonlinear Dynamic Viscoelasticity. Journal of Rheology, 23, 323-352. https://doi.org/10.1122/1.549518

[8] Briscoe, B., Luckham, P. and Zhu, S. (1998) Rheological Properties of Poly(ethylene oxide) Aqueous Solutions. Journal of Applied Polymer Science, 70, 419-429. https://doi.org/10.1002/(SICI)1097-4628(19981017)70:3<419::AID-APP1>3.0.CO;2$\underline{Q}$

[9] Coussot, P., Raynaud, J.S., Bertrand, F., Moucheront, P., Guilbaud, J.P., Huynh, H.T., Jarny, S. and Lesueur, D. (2002) Coeistence of Liquid and Solid Phases in 
Flowing Soft-Glassy Materials. Physical Review Letters, 88, Article ID: 218301. https://doi.org/10.1103/PhysRevLett.88.218301

[10] Niedzwiedz, K. (2008) Chain Dynamics and Viscoelastic Properties of Poly(ethylene-oxide). Macromolecules, 2008, 4866-4872.

https://doi.org/10.1021/ma800446n

[11] Guyon, E., Hulin, J.P. and Petit, L. (2001) Physical Hydrodynamics. EDP Sciences/CNRS Editions 2001.

[12] Couarraze, G. and Grossiord, J.L. (2000) Introduction to Rheology. 3rd Edition, Lavoisier.

[13] Riahi, M., Ouazzani, T. and Skali, L.S. (2017) Rheological Characterization of an Aqueous Solution of Polyethylene Oxide in Different Concentrations. 13th Congress of Mechanics, Meknes-Morocco, 11-14 April 2017, 129297.

[14] Berret, J.F. (2006) Rhéology of Worwlike Micelles: Equilibrium Properties and Shear-Banding Transitions. In: Weiss, R.G. and Terech, P., Eds., Molecular Gels. Materials with Self-Assembled Fibrillar Networks, The Journal of Physical Chemistry, Vol. 6, 667-720.

[15] Porte, G., Berret, J.F. and Harden, J. (1997) Inhomogenous Flows of Complex Fluids: Méchanical Instability versus Non Equilibrium Phase Transition. The European Physical Journal E, 7, 459-472.

[16] Spenley, N.A., Cates, M.E. and Meleiah, T.C. (1993) Nonlinear Rheology of Wormlikes Micelles. Physical Review Letters, 71, 939-942.

https://doi.org/10.1103/PhysRevLett.71.939

[17] Chedadi, I., Saramito, P. and Graner, F. (2012) Steady Couette Flows of Elastoviscoplastic Fluids Are Non-Unique. Journal of Rheology, 56, 213-239. https://doi.org/10.1122/1.3675605

[18] Barnes, H.A. (1999) The Yield Stress Everything Flows. Journal of Non-Newtonian Fluids Mechanics, 81, 133-178.

[19] Cheng, D.C.H. (1985) Yield Stress, a Time Defendant Property and How to Measure It. Rheologica Acta, 25, 542-554. https://doi.org/10.1007/BF01774406

[20] Picard, G. (2004) Heterogeneity of Threshold Fluid Flow: Phenomenological Approach and Elastoplastic Modeling. PhD Thesis, University of Paris VII, Denis-Dederot, Paris.

[21] Schurz, J. (1990) The Yield Stress-An Ampirical Reality. Rheological Acta, 29, 170-171. https://doi.org/10.1007/BF01332384

[22] Perge, C. (2013) French Congress of Mechanics Bordeaux, 26 to 30 August 2013, Bordeaux.

[23] Huang, N. (2005) Rheology of Granular Pastes. PhD Thesis, University Paris 6, Paris.

[24] Fall, A. (2008) Rheophysics of Complex Fluids: Flow and Blocking of Concentrated Suspensions. PhD Thesis, The University of Paris 7, Paris.

[25] Lami, S. (2015) Rheology and Flow of Suspension of Nano-Fibers Cellulose Fiber MRI Investigation in a Couette Device. 12th Congress of Mechanics, Casablanca-Morocco, 21-24 April 2015, 712-757.

[26] Rigal, C. (2012) Behavior of Complex Fluids under Flow: Experimental Approach by Nuclear Magnetic Resonance and Optical Techniques and Numerical Simulations. PhD Thesis, The University of Lorraine, Lorraine.

[27] Ovarlez, G. (2009) Phenomenology and Physical Origin of Shear-Localization and 
Shear-Banding in Complex Fluids. Rheologica Acta, 18, 831-844.

https://hal.archives-ouvertes.fr/hal-00454772 https://doi.org/10.1007/s00397-008-0344-6

[28] Guillemin, J.P. (2008) Rheology of Concentrated Suspensions of Recyclable Energy Materials: Modeling of the Casting Time. PhD Thesis, The National School of Mines of Saint Etienne, Sait Etienne.

[29] Ovarlez, G. (2011) Rheological Characterization of Threshold Fluids. Rhéol, 20, 28-43.

[30] Salmon, J.B., Manneville, S., Colin, A. and Molino, F. (2003) Velocity Proles in Shear-Banding Wormlike Micelles. Physical Review Letters, 90, Article ID: 228303. https://doi.org/10.1103/PhysRevLett.90.228303

[31] Bécu, L., Manneville, S. and Colin, A. (2004) Spatio-Temporal Dynamics of Wormlike Micelles under Shear. Physical Review Letters, 93, Article ID: 018301. https://doi.org/10.1103/PhysRevLett.93.018301

[32] Fardin, M.A. (2009) Taylor-Like Vortices in Shear Banding Flow of Giant Micelles. Physical Review Letters, 103, Article ID: 028302. https://doi.org/10.1103/PhysRevLett.103.028302

[33] Pakdel, P. and McKinley, G.H. (1996) Dynamics of Complex Fluids: Proceedings of the Second Royal Society-Unilever. Physical Review Letters, 77, 2459-2462. https://doi.org/10.1103/PhysRevLett.77.2459

[34] Ovarlez, G., Rodts, S., Château, X. and Coussot, P. (2009) Phenomenology and Physical Origin of Shear-Localization and Shear-Banding in Complex Fluids. Rheologica Acta, 48, 831-844. https://doi.org/10.1007/s00397-008-0344-6

[35] Fardin, M.A. (2009) Complex Fluid under Shear: Some Instabilities with Zero Reynolds Number. Physical Review Letters, 103, Article ID: 028302. https://doi.org/10.1103/PhysRevLett.103.028302

[36] Coussot, P. (2005) Rheometry of Pastes, Suspensions and Granular Materials. John Wiley \& Sons, Hoboken. https://doi.org/10.1002/0471720577

[37] Coussot, P. (2002-2004) Mechanical Characteristics and Origin of Wall Slip in Pasty Biosolids. Rheologica Acta, 43, 168-174.

[38] Gilbreth, S., Sullivan, S. and Dennin, M. (2006) Flow Transition in Two-Dimensional Foams. Physical Review E, 74, Article ID: 031401.

http://www.physics.uci.edu/ dennin/preprints/gsd06.pdf https://doi.org/10.1103/PhysRevE.74.051406

[39] Weaire, D., Barry, J.D. and Hutzler, S. (2010) The Continuum Theory of Shear Localization in Two-Dimensional Foam. Journal of Physics. Condensed Matter, 22, Article ID: 193101. https://doi.org/10.1088/0953-8984/22/19/193101

[40] Coussot, P., Nguyen, Q.D., Huynh, H.T. and Bonn, D. (2002) Avalanche Behavior in Yield Stress Fluids. Physical Review Letters, 88, Article ID: 175501. https://doi.org/10.1103/PhysRevLett.88.175501

[41] Groupe Français de Rhéologie (1993) Les cahiers de rhéologie. Vol. 11, numéro 1. 


\section{Nomenclature}

$r$. virtual cylinder position ( $\mathrm{mm}$ )

$r_{c}$ : flow and rest area interface $(\mathrm{mm})$

$R_{i}$ : radius of the virtual cylinder $(\mathrm{mm})$

$R_{1}$ : radius of the inner cylinder $(\mathrm{mm})$

$R_{2}:$ radius of the outer cylinder $(\mathrm{mm})$

$K$ : consistency $\left(\mathrm{N} \sec ^{2} / \mathrm{m}^{2}\right)$

$n$ : flow index

$V_{z}$ : axial component of the speed $(\mathrm{mm} / \mathrm{s})$

$V_{r}:$ radial component of velocity $(\mathrm{mm} / \mathrm{s})$

$V_{(\theta, r)}$ : tangential component of velocity $(\mathrm{mm} / \mathrm{s})$

$V_{0}$ : maximum tangential velocity expressed as a function of angular velocity $(\mathrm{mm} / \mathrm{s})$

$M$ : couple (Nm)

$R_{e}$ : Reynolds number

\section{Greek Letters}

$\dot{\gamma}$ : shear rate $\left(\mathrm{s}^{-1}\right)$

$\dot{\gamma}_{c}$ : critical shear rate $\left(\mathrm{s}^{-1}\right)$

$\tau$. shear stress $\mathrm{Pa}$

$\tau_{c}$ : Critical shear stress $(\mathrm{Pa})$

$\omega$ : rotation speed (rd./s)

$\omega_{c}:$ critical rotation speed ( $\left.\mathrm{rd} . / \mathrm{s}\right)$

$\sigma_{i}$ : shear stress at the virtual cylinder $(\mathrm{Pa})$

$\eta$ : viscosity $(\mathrm{Pa} \cdot \mathrm{s})$

$\mu$ : kinematic viscosity $\left(\mathrm{m}^{2} / \mathrm{s}\right)$

$\rho$ : density $\left(\mathrm{g} / \mathrm{cm}^{-3}\right)$

\section{Acronyms}

PEO: polyethylene oxide

\section{Indices}

C: critical point

1-2: index at point 1 , respectively 2

i: virtual point index 